\title{
Atlas of PET-CT: A Quick Guide to Image Interpretation
}

S. Fanti, M. Farsad, and L. Mansi, eds.

Berlin, Germany: Springer, 2009, 288 pages, $\$ 79.95$

This user-friendly, case-based book helps the reader to recognize normal, paraphysiologic, and pathologic uptake of ${ }^{18} \mathrm{~F}-\mathrm{FDG}$, methionine, and choline. About 500 PET/CT illustrations from 205 cases are presented. Each case not only shows PET findings but also includes some concise and useful teaching points.

The book is organized into 4 chapters covering the most relevant technical and pathophysiologic premises, clinical applications, and learning points and pitfalls. Chapter 1 has 17 cases dealing with normal distribution of ${ }^{18} \mathrm{~F}-\mathrm{FDG}$, which is used in more than $95 \%$ of PET procedures. Chapter 2 discusses 7 cases illustrating contrast-enhanced $\mathrm{CT}$ in PET/CT. Chapter 3 discusses 53 cases demonstrating the pitfalls of ${ }^{18} \mathrm{~F}-\mathrm{FDG}$ PET/CT: 8 of the cases are abdominal, 14 are bone, 8 are head and neck, 15 are chest, and 8 are pelvic. Chapter 4 illustrates 128 oncologic cases of the use of PET/CT for various organs and diseases. The oncologic cases used ${ }^{18} \mathrm{~F}-\mathrm{FDG}$, except for cases of brain and parathyroid tumors, which used methionine, and cases of prostate cancer, which used choline.
The images are clearly printed, and the teaching points are easily understood, especially through the use of arrows and color. Certain European terminology may not be familiar to American readers. European terms such as aspecific, miomatosis, mesogaster, oropharingeal, rhinopharynx, laterocervi$\mathrm{cal}$, and omolateral are used instead of their respective American counterparts: nonspecific, myomatosis, mesentery, oropharyngeal, nasopharynx, jugular, and ipsilateral. The subject index is somewhat limited, and there are no references.

This book aims to be a quick guide to interpreting PET/ CT images, obtained primarily with ${ }^{18} \mathrm{~F}-\mathrm{FDG}$, of oncologic patients. The book should be helpful to nuclear medicine and radiology practitioners whose work involves PET/CT, particularly those who are still learning about PET/CT. It will also be useful for both undergraduate and postgraduate students, as well as for course instructors.

E. Edmund Kim M.D. Anderson Cancer Center Houston, Texas 